Corrigendum

\title{
Corrigendum to "Ethyl Pyruvate Ameliorates Hepatic Ischemia-Reperfusion Injury by Inhibiting Intrinsic Pathway of Apoptosis and Autophagy"
}

\author{
Miao Shen, Jie Lu, Weiqi Dai, Fan Wang, Ling Xu, Kan Chen, Lei He, Ping Cheng, \\ Yan Zhang, Chengfen Wang, Dong Wu, Jing Yang, Rong Zhu, Huawei Zhang, \\ Yingqun Zhou, and Chuanyong Guo
}

Department of Gastroenterology, The Tenth People's Hospital of Tongji University, Shanghai 200072, China

Correspondence should be addressed to Yingqun Zhou; yqzh02@163.com and Chuanyong Guo; guochuanyong@hotmail.com

Received 20 March 2016; Accepted 26 May 2016

Copyright (C) 2016 Miao Shen et al. This is an open access article distributed under the Creative Commons Attribution License, which permits unrestricted use, distribution, and reproduction in any medium, provided the original work is properly cited.

In the article titled "Ethyl Pyruvate Ameliorates Hepatic Ischemia-Reperfusion Injury by Inhibiting Intrinsic Pathway of Apoptosis and Autophagy" [1], the name of the fifteenth author was given incorrectly as Yinqun Zhou. The author's name should have been written as Yingqun Zhou. The revised authors' list is shown above.

\section{References}

[1] M. Shen, J. Lu, W. Dai et al., "Ethyl pyruvate ameliorates hepatic ischemia-reperfusion injury by inhibiting intrinsic pathway of apoptosis and autophagy," Mediators of Inflammation, vol. 2013, Article ID 461536, 12 pages, 2013. 


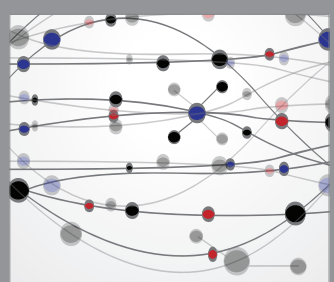

The Scientific World Journal
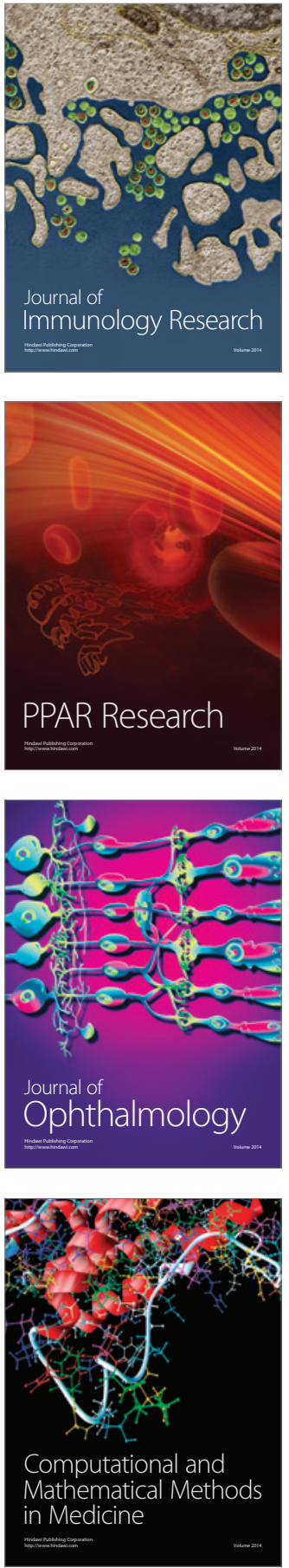

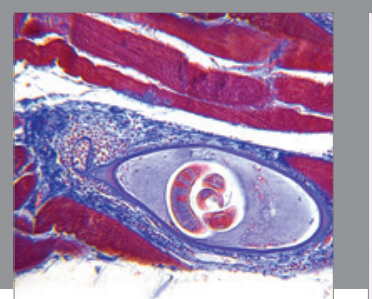

Gastroenterology Research and Practice

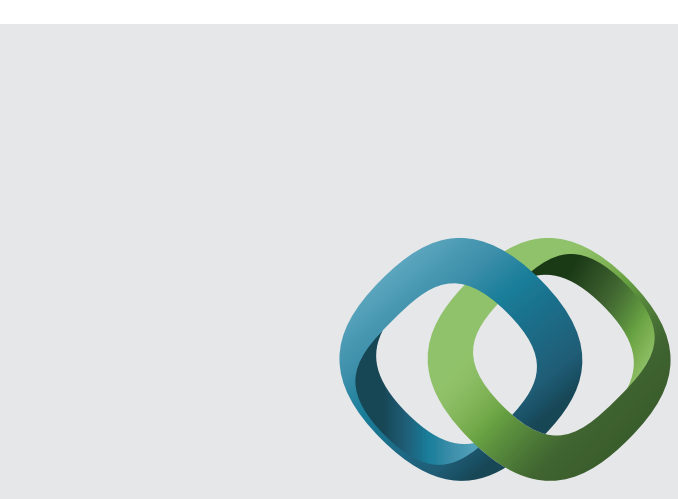

\section{Hindawi}

Submit your manuscripts at

http://www.hindawi.com
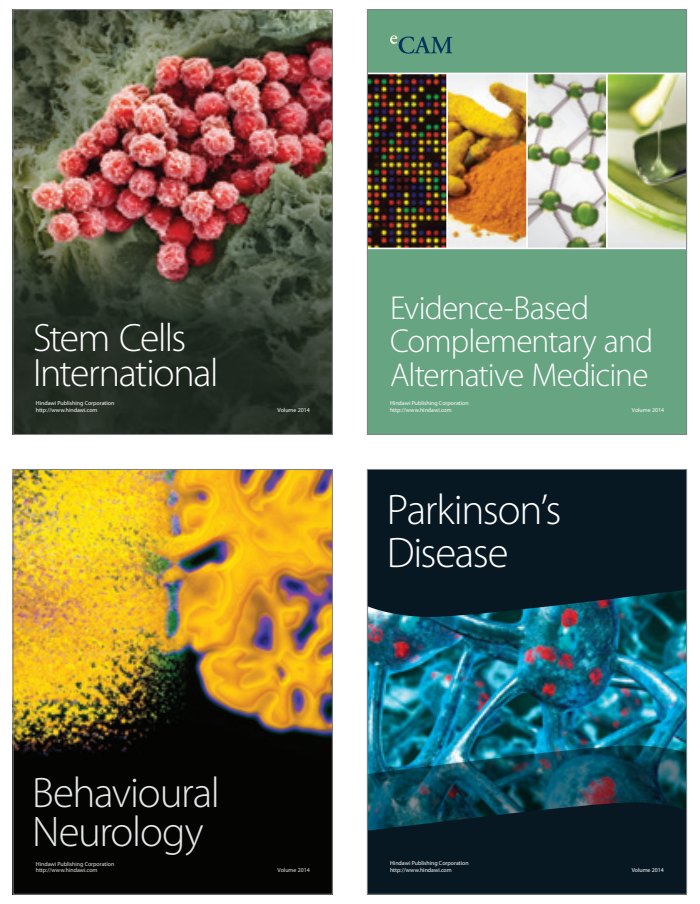
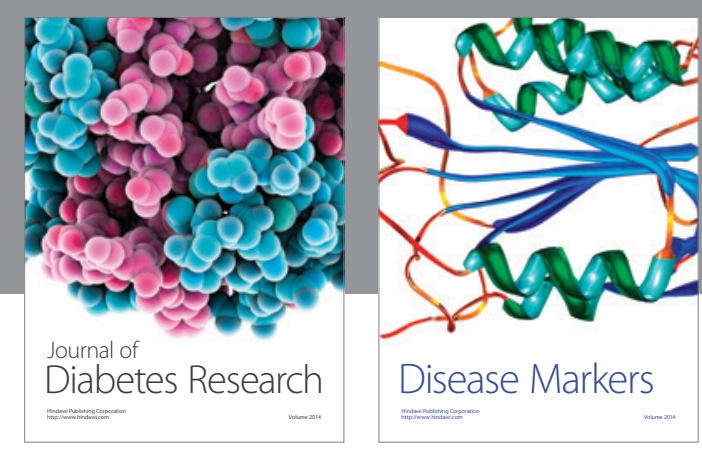

Disease Markers
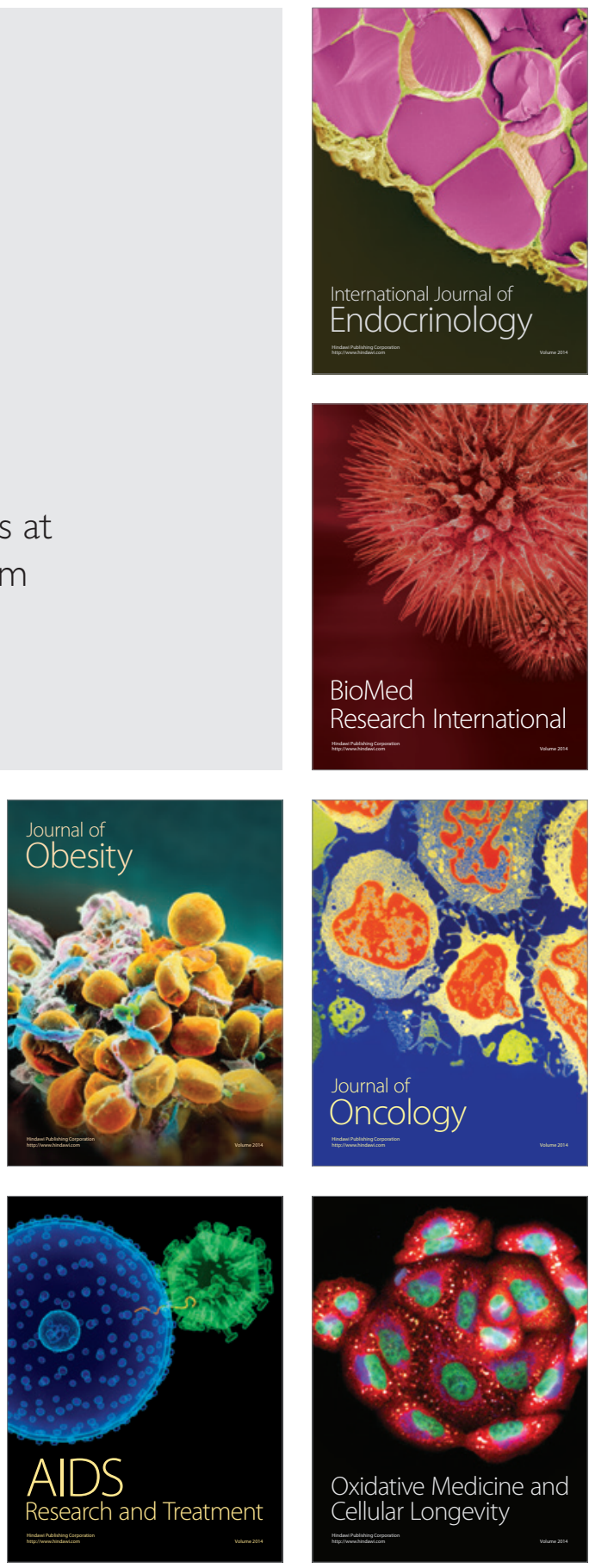